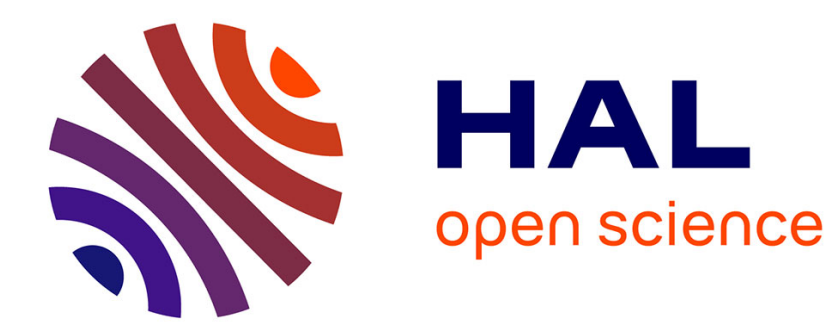

\title{
TENSOR-TRAIN DECOMPOSITION FOR IMAGE RECOGNITION *
}

D Brandoni, Valeria Simoncini

\section{To cite this version:}

D Brandoni, Valeria Simoncini. TENSOR-TRAIN DECOMPOSITION FOR IMAGE RECOGNITION *. 2019. hal-02196526

\section{HAL Id: hal-02196526 \\ https://hal.science/hal-02196526}

Preprint submitted on 28 Jul 2019

HAL is a multi-disciplinary open access archive for the deposit and dissemination of scientific research documents, whether they are published or not. The documents may come from teaching and research institutions in France or abroad, or from public or private research centers.
L'archive ouverte pluridisciplinaire HAL, est destinée au dépôt et à la diffusion de documents scientifiques de niveau recherche, publiés ou non, émanant des établissements d'enseignement et de recherche français ou étrangers, des laboratoires publics ou privés. 


\title{
TENSOR-TRAIN DECOMPOSITION FOR IMAGE RECOGNITION*
}

\author{
D. BRANDONI ${ }^{\dagger}$ AND V.SIMONCINI ${ }^{\ddagger}$
}

\begin{abstract}
We explore the potential of Tensor-Train (TT) decompositions in the context of multi-feature face or object recognition strategies. We devise a new recognition algorithm that can handle three or more way tensors in the TT format, and propose a truncation strategy to limit memory usage. Numerical comparisons with other related methods - including the well established recognition algorithm based on high-order SVD - illustrate the features of the various strategies on benchmark datasets.
\end{abstract}

1. Introduction. Automatic Object Classification and Face Recognition have become an important component in activities such as security and video-surveillance, where the quantities under scrutiny are the image pixels; see, e.g., [15] for some applications. Several factors, including illumination, view angle and expression, can affect the image, making the process of classification more complex. In the past, numerical linear algebra tools, and primarily the Singular Value Decomposition (SVD) have been extensively used to automatically process images in computations for classification purposes, see, e.g., [7] and its references. In this context, a database of $n_{p}$ persons in $n_{e}$ expressions is stored as $n_{p}$ distinct matrices $A_{p} \in \mathbb{R}^{n_{i} \times n_{e}}, p=1, \ldots, n_{p}$, where $n_{i}$ is the number of pixels of each image. SVD-based classification algorithms work pretty well when the number of image pixels is greater than the number of features, otherwise the classification performance drops significantly. This suggests that alternative strategies should be used, since the latter condition often occurs in realistic applications where very many images need to be analyzed.

More recently, it has been shown that multilinear algebra and the algebra of higher-order tensors (see, e.g., [10]) can offer a more powerful mathematical framework for analyzing and addressing the multi-factor structure of image ensembles. For instance, the Weizmann database in [18] - where TensorFaces are introduced - is composed by 28 male subjects in 15 poses, 4 illumination conditions and 3 expressions, and it is represented by a 5-way tensor. Then the High-Order SVD (HOSVD, see [5] and its references) is used to classify the image of an unknown person. Other tensorbased approaches have been proposed in a large variety of contexts; for instance, in [9] Face Recognition is performed using tensor-tensor decompositions, while in [2] the classification problem is expressed using the Kronecker Product Equation (KPE) in a randomization context. The very recent applied literature in object classification and face recognition methods is quite vast and it now extends to various machine learning strategies such as neural network ([19],[6]) and deep learning ([12]) methodologies. For these reasons, it is hard to clearly capture the algorithmic properties and specific features of the proposed methods, and at the same time it is difficult to single out a winning method among those recently proposed. Here we would like to limit our analysis to (algebraic) matrix- and tensor-based approaches, whose performance can be analyzed in a well established manner.

We explore the use of the Tensor-Train Decomposition (TT-Decomposition) for multi-feature recognition strategies. This tensor decomposition has been shown to

\footnotetext{
*This version dated July 21, 2019.

$\dagger$ Dipartimento di Matematica Università di Bologna, Piazza di Porta San Donato 5, Bologna, Italy (domitilla.brandoni2@unibo.it).

${ }_{\ddagger}^{\ddagger}$ Dipartimento di Matematica Università di Bologna, Piazza di Porta San Donato 5, Bologna, and IMATI-CNR, Pavia (valeria.simoncini@unibo.it).
} 
have great potential in multivariate function approximation and compression, and it is particularly amenable to truncation strategies yielding low-rank tensor approximations. The decomposition unwraps the given multi-way tensor in an appropriate chain ("train") of three-dimensional tensors and it is thus appropriate to handle high order tensors, as opposed to HOSVD, which requires to allocate memory for a core tensor with the same order of $\mathcal{A}$. We have thus devised a recognition algorithm based on the TT Decomposition, which extends the recognition method of HOSVD to this setting; to the best of our knowledge this algorithm is new.

A synopsis of the paper is as follows. In section 2 we recall some standard properties of tensor computations, that will be used throughout the paper. In section 3 we present the TT-form of the given image-related tensor. With section 4 we start our discussion of classification algorithms, beginning with a simple minded least-squares method, and then relating it to the algorithm based on the HOSVD. In subsection 4.3 we introduce our TT classification algorithm for the 3D case, making some algebraic comparisons with known methods and discussing a truncation strategy. In section 5 we extend our algorithm to fourth (or higher) order tensors. In section 6 we present the eight databases, which will be used to exercise the various algorithms in section 7 using different performance measures. We include our conclusions in section 8 .

2. Tensor Computations. In this section we summarize some of the basic facts about tensors and their computations, that will be used in the paper.

A higher-order tensor is a multidimensional array, corresponding to an element of a tensor product of $N$ vector spaces. First-order tensors (that is, vectors) are usually denoted by lowercase letters $(a, b, c, \ldots)$, second-order tensors (matrices) are denoted by capital letters $(A, B, C, \ldots)$ and higher-order tensors are denoted by calligraphic letters $(\mathcal{A}, \mathcal{B}, \mathcal{C}, \ldots)$. The order of a tensor is the number of dimensions, also known as ways or modes. For example, a tensor $\mathcal{A} \in \mathbb{R}^{4 \times 2 \times 3}$ is a third-order tensor.

Two important tensor operations need to be introduced: the $n$-mode product, which is a tensor by matrix operation, and the $\left(\begin{array}{c}m \\ n\end{array}\right)$-product, which is a tensor-bytensor operation.

Definition 2.1. [10, p. 460] Given the tensor $\mathcal{A} \in \mathbb{R}^{I_{1} \times I_{2} \times \cdots \times I_{N}}$, the n-mode matrix product of $\mathcal{A}$ with a matrix $U \in \mathbb{R}^{J \times I_{n}}$ is denoted by

$$
\mathcal{A} \times{ }_{n} U \in \mathbb{R}^{I_{1} \times \cdots \times I_{n-1} \times J \times I_{n+1} \times \cdots \times I_{N}}
$$

and its entries are given by

$$
\left(\mathcal{A} \times{ }_{n} U\right)_{i_{1}, \ldots, i_{n-1}, j, i_{n+1}, \ldots, I_{N}}=\sum_{i_{n}=1}^{I_{n}} a_{i_{1}, i_{2}, \ldots, i_{n}, \ldots, i_{N}} u_{j, i_{n}}
$$

The $n$-mode multiplication satisfies the following important commutativity property,

$$
\mathcal{A} \times{ }_{n} U \times_{m} V=\mathcal{A} \times{ }_{m} V \times_{n} U \quad \forall m \neq n,
$$

where $\mathcal{A} \in \mathbb{R}^{I_{1} \times \cdots \times I_{n} \times \cdots \times I_{m} \times \cdots \times I_{N}}, U \in \mathbb{R}^{J \times I_{n}}$ and $V \in \mathbb{R}^{J \times I_{m}}$. Therefore, in a series of multiplications the product order of distinct modes is irrelevant. If the modes are the same, i.e. $m=n$, then

$$
\mathcal{A} \times_{n} U \times_{n} V=\mathcal{A} \times_{n}(V U) .
$$

In a similar way to the $n$-mode product, in [4] the $\left(\begin{array}{c}m \\ n\end{array}\right)$-product of a tensor is introduced. 
Definition 2.2. [4] The $\left(\begin{array}{c}m \\ n\end{array}\right)$-product of a tensor $\mathcal{A} \in \mathbb{R}^{I_{1} \times I_{2} \times \cdots \times I_{N}}$ with a tensor $\mathcal{B} \in \mathbb{R}^{J_{1} \times J_{2} \times \cdots \times J_{M}}$, such that $I_{n}=J_{m}$, is defined as

$$
\mathcal{C}=\mathcal{A} \times{ }_{n}^{m} \mathcal{B}
$$

where $\mathcal{C} \in \mathbb{R}^{I_{1} \times \cdots \times I_{n-1} \times I_{n+1} \times \cdots \times I_{N} \times J_{1} \times \cdots \times J_{m-1} \times J_{m+1} \times \cdots \times J_{M}}$. Its entries are given by

$$
\begin{aligned}
\mathcal{C}\left(i_{1}, \ldots, i_{n-1}, i_{n+1}, \ldots, i_{N}, j_{1}, \ldots j_{m-1}, j_{m+1}, \ldots, j_{M}\right) & = \\
& \sum_{i=1}^{I_{n}} \mathcal{A}\left(i_{1}, \ldots, i_{n-1}, i, i_{n+1}, \ldots, i_{N}\right) \mathcal{B}\left(j_{1}, \ldots, j_{m-1}, i, j_{m+1}, \ldots, j_{M}\right) .
\end{aligned}
$$

Finally, we define the process of flattening a tensor into a matrix, i.e. the unfolding. There are several ways to unfold a tensor into a matrix. In this work we refer to the one used by Oseledets in [13]. Let $\mathcal{A} \in \mathbb{R}^{I_{1} \times I_{2} \times \cdots \times I_{N}}$ be an $N$ th-order tensor. The $k$-th unfolding of $\mathcal{A}$ is a matrix $A_{k} \in \mathbb{R}^{\left(I_{1} I_{2} \ldots I_{k}\right) \times\left(I_{k+1} I_{k+2} \ldots I_{N}\right)}$, whose elements are taken column-wise from $\mathcal{A}$, that is

$$
A_{k}\left(i_{1} \cdot \ldots \cdot i_{k}, i_{k+1} \cdot \ldots \cdot i_{N}\right)=\mathcal{A}\left(i_{1}, \ldots, i_{k}, i_{k+1}, \ldots i_{N}\right) .
$$

3. Tensor-Train Decomposition. The Tensor-Train Decomposition factorizes an $N$ th-order tensor $\mathcal{A}$ in a product of third-order tensors and it is given by ([13])

$$
\begin{aligned}
\mathcal{A}\left(i_{1}, \ldots, i_{N}\right) & =G_{1}\left(i_{1},:\right) \mathcal{G}_{2}\left(:, i_{2},:\right) \mathcal{G}_{3}\left(:, i_{3},:\right) \ldots G_{N}\left(i_{N},:\right) \\
& =G_{1}\left(i_{1}\right) \mathcal{G}_{2}\left(i_{2}\right) \mathcal{G}_{3}\left(i_{3}\right) \ldots G_{N}\left(i_{N}\right)
\end{aligned}
$$

where $G_{1}, \mathcal{G}_{2}, \ldots, G_{N}$ are called $T T$-cores.

In index form, the definition (3.1) can be written as

$$
\mathcal{A}\left(i_{1}, \ldots, i_{N}\right)=\sum_{\alpha_{1}, \ldots, \alpha_{N-1}} G_{1}\left(i_{1}, \alpha_{1}\right) \mathcal{G}_{2}\left(\alpha_{1}, i_{2}, \alpha_{2}\right) \ldots G_{N}\left(\alpha_{N-1}, i_{N}\right) .
$$

A classical visualization of the decomposition of a third-order tensor in index form is given in Figure 3.1 (see [13]). In this work we consider the TT-SVD decomposition,

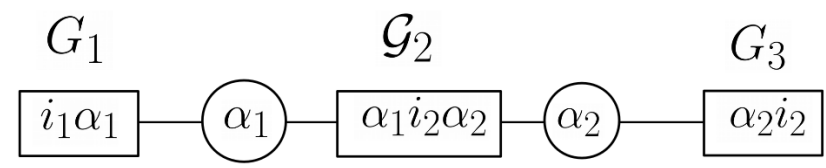

Fig. 3.1: Visualization of the Tensor Train Decomposition in index form.

i.e. each factor of the Tensor-Train Decomposition is computed using the SVD of a specific unfolding of $\mathcal{A}$. For example, the first term of the decomposition is obtained by considering the SVD of $A_{1}=G_{1} \Sigma_{1} V_{1}^{T}$. For further details we refer the reader to the seminal work [13] and to [17].

Using Definition 2.2 for the $\left(\begin{array}{c}m \\ n\end{array}\right)$-mode product, (3.2) can be written as

$$
\mathcal{A}=G_{1} \times{ }_{2}^{1} \mathcal{G}_{2} \times{ }_{3}^{1} \mathcal{G}_{3} \times{ }_{3}^{1} \cdots \times{ }_{3}^{1} G_{N} .
$$


Thus, if $\mathcal{A}$ is a third-order tensor, then

$$
\mathcal{A}=\mathcal{G}_{2} \times{ }_{1} G_{1} \times{ }_{3} G_{3}^{T},
$$

since

$$
\begin{aligned}
\mathcal{A}\left(i_{1}, i_{2}, i_{3}\right) & =G_{1}\left(i_{1},:\right) \times{ }_{2}^{1} \mathcal{G}_{2}\left(:, i_{2},:\right) \times{ }_{3}^{1} G_{3}\left(:, i_{3}\right)=\sum_{\alpha_{1}, \alpha_{2}} G_{1}\left(i_{1}, \alpha_{1}\right) \mathcal{G}_{2}\left(\alpha_{1}, i_{2}, \alpha_{2}\right) G_{3}\left(\alpha_{2}, i_{3}\right) \\
& =\sum_{\alpha_{2}}\left(\mathcal{G}_{2} \times{ }_{1} G_{1}\right)\left(i_{1}, i_{2}, \alpha_{2}\right) G_{3}\left(\alpha_{2}, i_{3}\right)=\left(\mathcal{G}_{2} \times{ }_{1} G_{1} \times{ }_{3} G_{3}^{T}\right)\left(i_{1}, i_{2}, i_{3}\right) .
\end{aligned}
$$

A standard visualization of (3.4) is given in Figure 3.2.

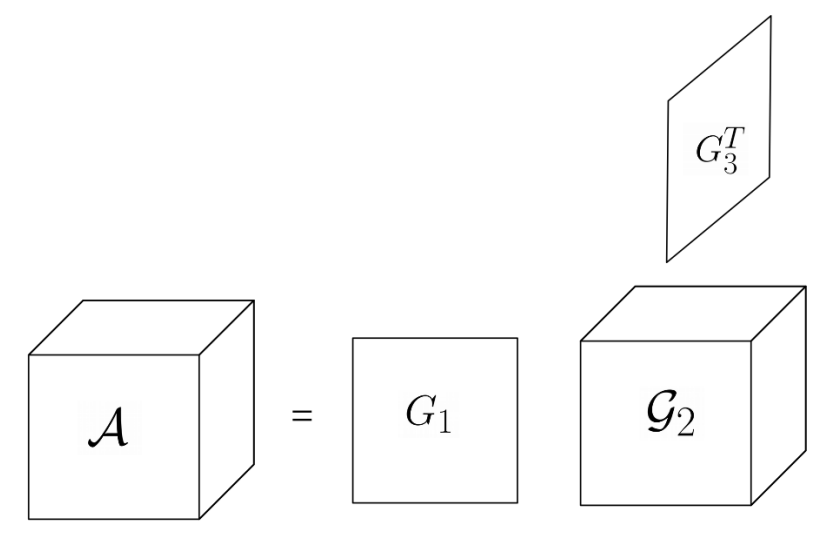

Fig. 3.2: Visualization of the Tensor Train Decomposition of a third-order tensor using the $\left(\begin{array}{c}m \\ n\end{array}\right)$-mode product.

To make tensor computations affordable, truncation is commonly employed, as classically performed in the matrix SVD setting; we refer the reader to, e.g., [9],[3],[14] where tensor truncations are discussed. We thus consider a truncated version of the TT-SVD of $\mathcal{A}$, exploiting the singular value decay of $A_{1}$ (in Figure 3.3 we report one such pattern for one of the considered databases). In our setting we decided to truncate only along the first mode, however, the same procedure can be used to perform the truncation also along the second and third modes. We refer to, e.g., [11] for a discussion on feasible singular values. In our truncation strategy we retain the first $k$ singular values of $A_{1}$ whose sum provides the $\pi 100 \%$ of the total singular value sum. Thus, we consider only the first $k$ columns of $G_{1}$, and name the resulting matrix $\hat{G_{1}}$. As a consequence, we also set $\hat{\mathcal{G}_{2}} \in \mathbb{R}^{k \times n_{e} \times n_{p}}$ as the tensor of the first $k$ first-mode elements of $\mathcal{G}_{2}$. Hence the truncated TT-SVD decomposition has the following form

$$
\mathcal{A}=\hat{\mathcal{G}_{2}} \times{ }_{1} \hat{G_{1}} \times{ }_{3} G_{3}^{T} .
$$

The error due to this truncation can be estimated as described in [13].

4. 3D Classification problem and algorithms. In this and the next sections we recall some known tensor-based classification algorithms, and we introduce a classification strategy associated with the Tensor-Train decomposition. In this first section 


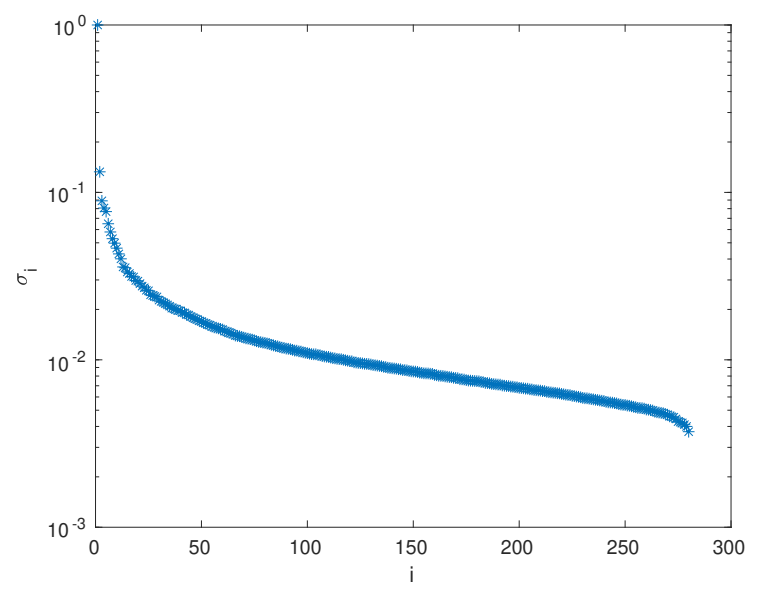

Fig. 3.3: Singular values of $A_{1}$ for the Orl Database.

we consider the TT decomposition of a 3D tensor, while we postpone to section 5 that of a $4 \mathrm{D}$ tensor, anticipating that the TT formulation allows us to treat the two cases in exactly the same manner. To the best of our knowledge, the use of these strategies in the classification context is new.

Let $\mathcal{A} \in \mathbb{R}^{n_{i} \times n_{e} \times n_{p}}$ be the tensor representing the database of images with $n_{i}$ pixels, $n_{p}$ persons and $n_{e}$ expressions for each person. Given an image $z$ of an unknown person in an unknown expression, represented by a vector in $\mathbb{R}^{n_{i}}$, we want to determine which of the $n_{p}$ persons, the new image is closest to. To this end we define a distance $\operatorname{dist}(z, \mathcal{A}(:,:, p))$ of $z$ from each person $p$ of the given database, for $p=1, \ldots, n_{p}$.

In the following discussion $\mathbf{E}^{e}$ denotes the space "expression $e$ ". This space is spanned by all images of the $n_{p}$ persons in expression $e$.

4.1. Least Squares Classification Algorithm. This simple minded classification strategy merely focuses on the matrices obtained by comparing with respect to all images in each given expression. More precisely, for each expression $e$ let $A^{e}:=\mathcal{A}(:, e,:)$, where the columns of $A^{e}$ have been scaled to have unit Euclidean norm. Consider the following least squares problem

$$
\min _{x}\left\|z-A^{e} x\right\|_{2}
$$

Let $x_{e}$ be the solution to (4.1), and let $r_{e}=z-A^{e} x_{e}$ be the associated residual. The distance of $z$ from $\mathbf{E}^{e}$ is obtained by $\left\|r_{e}\right\|_{2}$. We then compute

$$
\hat{e}=\operatorname{argmin}_{e}\left\|r_{e}\right\|_{2}
$$

and we classify $z$ as person $\hat{p}$, where $\hat{p}=\operatorname{argmax}_{p}\left|x_{\hat{e}}(p)\right|$, that is $\hat{p}$ corresponds to the largest component of the $x_{\hat{e}}$, where $x_{\hat{e}}$ holds the linear combination of all persons in the closest expression. A version of the classification algorithm is given in Algorithm 4.1.

\footnotetext{
${ }^{1}$ Each slice of $\mathcal{A}$, that is $A^{e}$, is normalized before solving (4.1).
} 


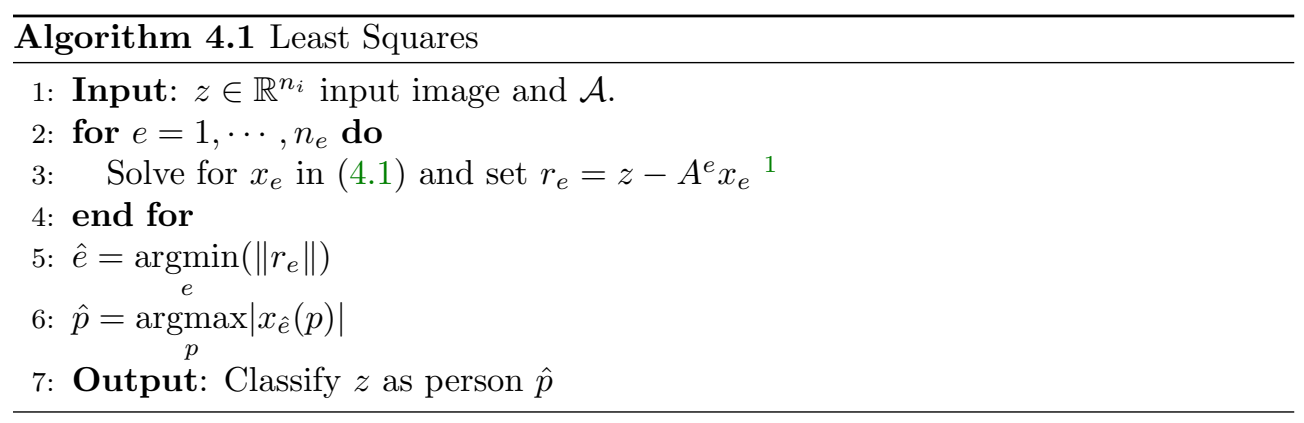

4.2. HOSVD Classification Algorithm. Using the HOSVD described in [5], the tensor $\mathcal{A} \in \mathbb{R}^{n_{i} \times n_{e} \times n_{p}}$ can be written as follows

$$
\mathcal{A}=\mathcal{S} \times{ }_{i} F \times{ }_{e} G \times{ }_{p} H
$$

where $\times_{i}, \times_{e}, \times_{p}$ are the 1-mode, 2-mode, 3-mode multiplications, respectively ${ }^{2}$; with $G \in \mathbb{R}^{n_{e} \times n_{e}}, H \in \mathbb{R}^{n_{p} \times n_{p}}$ and, if $n_{i}>n_{e} n_{p}$ then $\mathcal{S} \in \mathbb{R}^{n_{e} n_{p} \times n_{e} \times n_{p}}, F \in \mathbb{R}^{n_{i} \times n_{e} n_{p}}$, otherwise, $\mathcal{S} \in \mathbb{R}^{n_{i} \times n_{e} \times n_{p}}, F \in \mathbb{R}^{n_{i} \times n_{i}}$.

Letting $\mathcal{C}=\mathcal{S} \times{ }_{i} F \times{ }_{e} G$, for a fixed expression $e$

$$
A^{e}=C^{e} H^{T} \quad e=1,2, \ldots, n_{e},
$$

where $A^{e}$ and $C^{e}$ are the matrices obtained by fixing the second index of $\mathcal{A}$ and $\mathcal{C}$ equal to $e$. Note that the $n_{p} \times n_{p}$ matrix $H^{T}$ is orthogonal and that the matrix $C^{e}$ is $n_{i} \times n_{p}$. Column $p$ of $A^{e}$ can be written as

$$
a_{p}^{(e)}=C^{e} h_{p}^{T},
$$

where $h_{p}^{T}$ is the $p$-th column of $H^{T}$. Now let $z \in \mathbb{R}^{n_{i}}$ be the new image to be classified. The coordinates of $z$ in the expression basis can be found by solving a least squares problem

$$
\hat{\alpha}_{e}=\operatorname{argmin}_{\alpha_{e}}\left\|C^{e} \alpha_{e}-z\right\|_{2}, \quad e=1, \cdots, n_{e} .
$$

Then, for each $e=1, \cdots, n_{e}$ and for each $p=1, \cdots, n_{p}$

$$
D_{H O}(e, p)=\left\|\hat{\alpha_{e}}-h_{p}^{T}\right\|_{2} .
$$

Note that if $C^{e}$ is full column rank, then the least squares problem (4.4) has a unique solution, otherwise the solution is not unique, but the solution with smallest norm can be obtained by using the SVD of $C^{e}$ for its computation ([8, section 5.5]). A version of the classification algorithm based on the HOSVD can be found in [7, Chapter 14, p.174], and this is what was used in our experiments.

There is a tight connection between the LS-based method and the HOSVD classification, given that for each expression $e$ the matrix $A^{e}$ is the same.

Proposition 4.1. Let $x_{e}$ be the solution to (4.1) in the LS-based method, and let $\hat{\alpha}_{e}$ be the solution to (4.4) in the HOSVD method. Then $\hat{\alpha}_{e}=H^{T} x_{e}$. Letting $I_{:, p}$ be the $p$-th column of the canonical basis, it then holds

$$
D_{H O}(e, p)=\left\|\hat{\alpha_{e}}-h_{p}^{T}\right\|_{2}=\left\|x_{e}-I_{:, p}\right\|_{2} .
$$

\footnotetext{
${ }^{2} \times_{i}$ is the image-mode multiplication, $\times_{e}$ is the expression-mode multiplication, $\times_{p}$ is the personmode multiplication.
} 


\begin{tabular}{|c|c|c|c|c|}
\hline Database & $p$ & LS & LS2 & HOSVD \\
\hline Orl & 14 & 18 & 14 & 14 \\
\hline COIL-20 & 1 & 1 & 1 & 1 \\
\hline Faces95 & 5 & 50 & 57 & 57 \\
\hline Faces96 & 59 & 77 & 59 & 59 \\
\hline Ext'd Yale shrunk & 9 & 10 & 6 & 6 \\
\hline
\end{tabular}

Table 4.1: Classification performance of least squares based algorithms and the algorithm based on HOSVD.

Proof. The least squares solution $\hat{\alpha}_{e}$ is given by $\hat{\alpha}_{e}=\left(\left(C_{e}\right)^{T} C_{e}\right)^{-1}\left(C_{e}\right)^{T} z$, while the least squares solution $x_{e}$ is given by $x_{e}=\left(\left(A^{e}\right)^{T} A^{e}\right)^{-1}\left(A^{e}\right)^{T} z$. Substituting $A^{e}=C^{e} H^{T}$ we obtain

$$
\begin{aligned}
x_{e} & =\left(\left(A^{e}\right)^{T} A^{e}\right)^{-1}\left(A^{e}\right)^{T} z=\left(H\left(C^{e}\right)^{T} C^{e} H^{T}\right)^{-1} H\left(C^{e}\right)^{T} z \\
& =H^{-T}\left(\left(C^{e}\right)^{T} C^{e}\right)^{-1}\left(C^{e}\right)^{T} z=H^{-T} \hat{\alpha}_{e},
\end{aligned}
$$

which proves the first assertion. For the second assertion, since $\hat{\alpha}_{e}=H^{T} x_{e}$, we have $\left\|\hat{\alpha_{e}}-h_{p}^{T}\right\|_{2}=\left\|H^{T} x_{e}-H^{T} I_{:, p}\right\|_{2}=\left\|H^{T}\left(x_{e}-I_{:, p}\right)\right\|_{2}$. From the orthogonality of $H$ the result follows.

Assume that $z$ and $A^{e}$ have both been scaled to have unit norm columns, so that the entries of $x_{e}$ are all not greater than one in absolute value. It follows that for a fixed $e$, finding the minimum of $D_{H O}(e, p)$ corresponds to finding the largest entry of $x_{e}$, the $\hat{p}$-th entry, which is closest to one. Hence, the difference between the two methods lays in the way the "best" expression is chosen. In the basic least squares method the expression corresponding to the smallest residual norm is selected, whereas in HOSVD all is based on the quantity $\left\|x_{e}-I_{:, p}\right\|$. This implies that the HOSVD and LS methods compute the same quantities, but the stopping criterion changes, which is thus responsible of possible discrepancies in the performance of the two approaches (see, e.g., the results for the Fashion MNIST dataset).

Table 4.1 displays the differences and similarities between the least-squares and the HOSVD formulations for the classification of a single image: the test set is composed by the person $p$ in the last expression, while all the other $n_{p}$ persons in the remaining expressions are used as training set. We report the classification result of each method by displaying $\hat{p}$ for each database. The method LS2 corresponds to Algorithm 4.1, in which lines 5 and 6 are replaced with $[\hat{e}, \hat{p}]=\operatorname{argmin} D(e, p)=\operatorname{argmin}\left\|x_{e}-I_{:, p}\right\|$. According to Proposition 4.1, the same classification results are obtained using either the HOSVD or LS2.

4.3. Tensor-Train Classification Algorithm. To derive the new classification algorithm we first write the data tensor for each expression $e$, by means of a TT-based basis for $\mathbf{E}^{e}$. Then we define the associated distance to be minimized.

Using (3.4), $\mathcal{A}(:, e,:)$ can be written as

$$
\mathcal{A}(:, e,:)=G_{2}^{e} \times{ }_{i} G_{1} \times{ }_{p} G_{3}^{T} \quad \forall e=1, \ldots, n_{e},
$$

where $G_{2}^{e}=\mathcal{G}_{2}(:, e,:)$ is a matrix. Thus, the image of a person $p$ in the expression $e$ is

$$
\mathcal{A}(:, e, p)=G_{1} G_{2}^{e} g_{3}^{p}, \quad \text { where } \quad g_{3}^{p}=G_{3}(:, p) .
$$


To deal with the classification problem, it is better to set $\mathcal{C}=\mathcal{G}_{2} \times_{i} G_{1}$ so that (4.6) becomes

$$
\mathcal{A}(:, e, p)=C^{e} g_{3}^{p}, \quad \text { with } \quad C^{e}=\mathcal{C}(:, e,:)=G_{1} G_{2}^{e} .
$$

This can be interpreted as follows. The columns of $C^{e}$ are a basis for $\mathbf{E}^{e}$ while $g_{3}^{p}$, the $p$-th column of $G_{3}$, holds the coordinates of person $p$ in this basis. Notice that the same $g_{3}^{p}$ holds the coordinates of all the images of person $p$ in the different expression bases [7, p.173]. The expression above plays the same role as (4.3) for the HOSVD.

Given the image $z$ of an unknown person in an unknown expression, we want to find the coordinates $\alpha_{e}$ of $z$ in all the $n_{e}$ bases $\left\{C^{e}\right\}_{e=1, \ldots, n_{e}}$ and then compare each $\alpha_{e}$ for $e=1, \ldots, n_{e}$ with the coordinates of all $n_{p}$ persons in the same basis, which are represented by the columns of $G_{3}$. More precisely, for each $e=1, \cdots, n_{e}$ we compute

$$
\min _{\alpha_{e}}\left\|C^{e} \alpha_{e}-z\right\|_{2},
$$

and then, for each $e=1, \cdots, n_{e}$ and for each $p=1, \cdots, n_{p}$

$$
D_{T T}(e, p):=\left\|\hat{\alpha_{e}}-g_{3}^{p}\right\|_{2} .
$$

Hence, the distance between $z$ and person $p$ is given by

$$
\operatorname{dist}(z, \mathcal{A}(:,:, p))=\min _{e} D_{T T}(e, p) .
$$

The computation in (4.8) can be performed by means of the reduced QR decomposition of $G_{2}^{e}$, that is $G_{2}^{e}=Q^{e} R^{e}$. The coordinates of $z$ in $\mathbf{E}^{e}$ are thus given by $\hat{\alpha_{e}}=R^{(e)^{-1}} Q^{(e)^{T}} G_{1}^{T} z$ and so (4.9) becomes

$$
D_{T T}(e, p)=\left\|R^{(e)^{-1}} Q^{(e)^{T}} G_{1}^{T} z-g_{3}^{p}\right\|_{2} \quad p=1, \cdots, n_{p},
$$

where $g_{3}^{p}=G_{3}(:, p)$. The overall procedure is summarized in Algorithm 4.2.

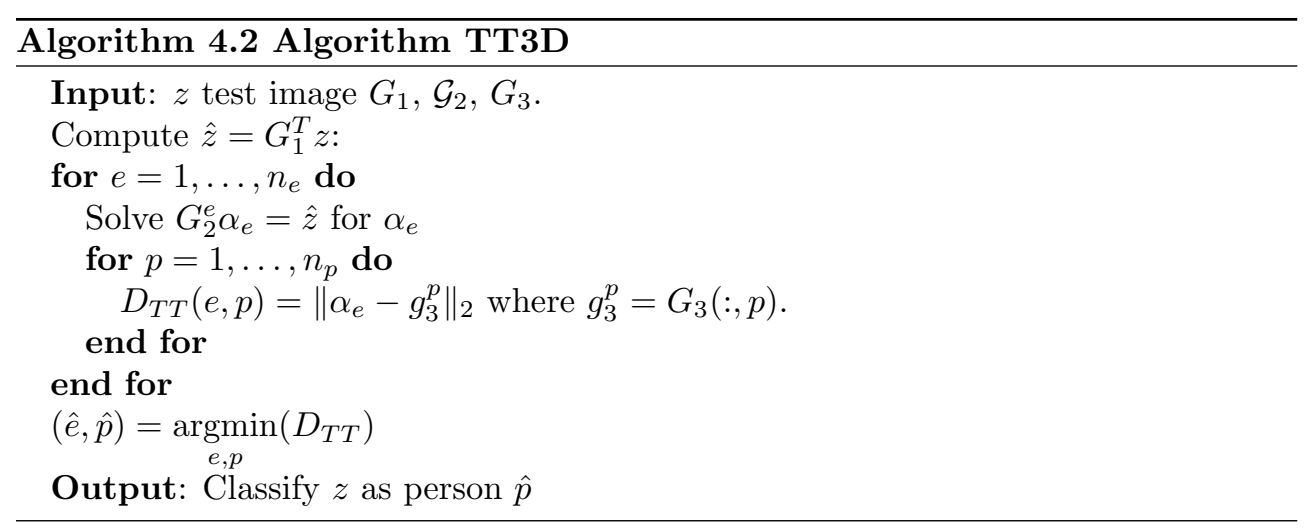

REMARK 4.2. Let $A^{e}=\mathcal{A}(:, e,:)$ in (4.6). Then a result analogous to that in Proposition 4.1 can be derived, that is the distance $D_{T T}$ satisfies

$$
D_{T T}(e, p)=\left\|x_{e}-I_{:, p}\right\|
$$

where $x_{e}=\operatorname{argmin}_{x}\left\|A^{e} x-z\right\|$. 
5. 4D Classification algorithm with Tensor-Train decomposition. Several authors in the literature have observed that image classification can be improved by considering images as matrices $I \in \mathbb{R}^{n_{1} \times n_{2}}$ instead of vectors, thus adding a forth dimension to the problem; see, e.g., [9]. Hence, a database of $n_{p}$ persons in $n_{e}$ expressions is represented by a four-dimensional tensor $\mathcal{A} \in \mathbb{R}^{n_{1} \times n_{2} \times n_{e} \times n_{p}}$, written in TT-form as

$$
\mathcal{A}=G_{1} \times{ }_{2}^{1} \mathcal{G}_{2} \times{ }_{3}^{1} \mathcal{G}_{3} \times{ }_{3}^{1} G_{4},
$$

where $G_{1} \in \mathbb{R}^{n_{1} \times n_{1}}, \mathcal{G}_{2} \in \mathbb{R}^{n_{1} \times n_{2} \times n_{e} n_{p}}, \mathcal{G}_{3} \in \mathbb{R}^{n_{e} n_{p} \times n_{e} \times n_{p}}$, and $G_{4} \in \mathbb{R}^{n_{p} \times n_{p}}$. Thus, the image of a person $p$ in expression $e$ in the database is given by

$$
\mathcal{A}(:,:, e, p)=\mathcal{G}_{2} \times{ }_{1} G_{1} \times{ }_{3}\left(G_{3}^{(e)}\right)^{T} \times_{3} g_{4}^{(p)},
$$

where $G_{3}^{(e)}=\mathcal{G}_{3}(:, e,:) \in \mathbb{R}^{n_{e} n_{p} \times n_{p}}$ and $g_{4}^{(p)}=G_{4}(:, p) \in \mathbb{R}^{n_{p}}$. Let $\mathcal{C}^{(e)}=\mathcal{G}_{2} \times_{1} G_{1} \times{ }_{3}$ $\left(G_{3}^{(e)}\right)^{T}$. Then (5.1) becomes

$$
\mathcal{A}(:,:, e, p)=\mathcal{C}^{(e)} \times{ }_{3} g_{4}^{(p)} .
$$

The classification strategy is then analogous to that for the three-dimensional case. In particular, given an image $z \in \mathbb{R}^{n_{1} n_{2}}$ of an unknown person in an unknown expression, we define the distance of $z$ from person $p$ as

$$
D_{T T 4}(e, p)=\left\|\hat{\alpha_{e}}-g_{4}^{(p)}\right\|_{2},
$$

where

$$
\hat{\alpha_{e}}=\operatorname{argmin}_{\alpha_{e}}\left\|\operatorname{unfold}\left(\mathcal{C}^{(e)}\right) \alpha_{e}-z\right\|_{2} .
$$

A version of the classification algorithm is given in Algorithm TT 4D. In our experiments, we used the unfolding tenmat $\left(\mathcal{C}^{e}, 3\right.$, 't') from the Matlab Tensor Toolbox [1].

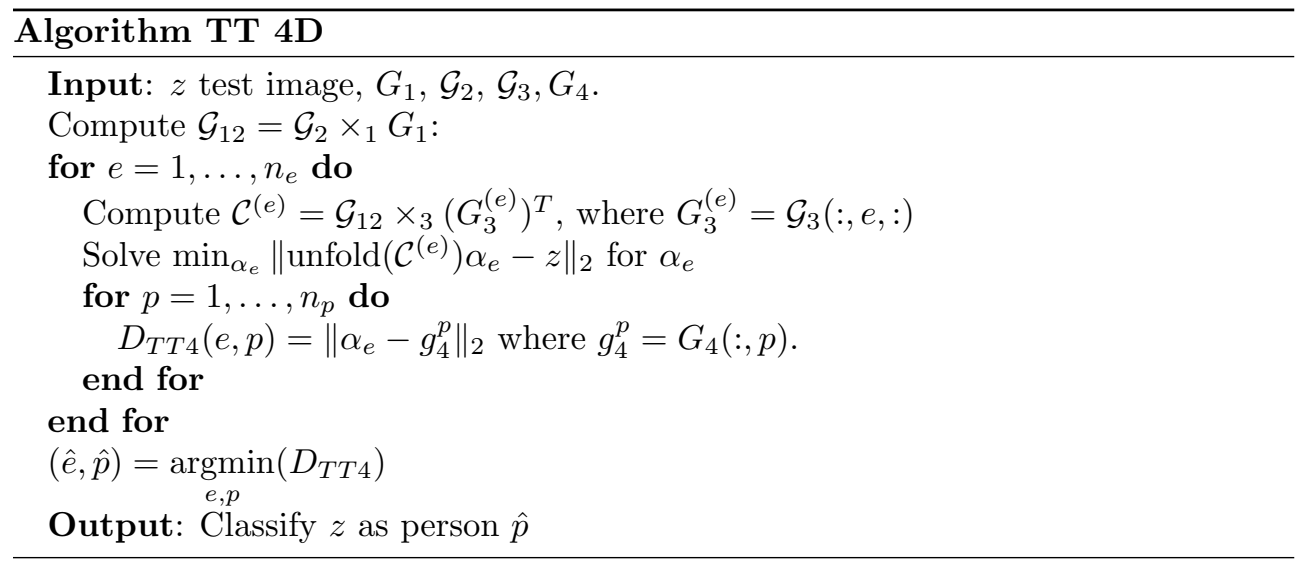

The TT format naturally extends to the higher-dimensional setting. For instance, suppose that $\mathcal{A}$ is an $N$ th order tensor representing our database, further assume that the first two modes are the pixel modes (as in TT4D) and the last one is the person 
mode. According to (3.3) $\mathcal{A}$ can be written in the following way:

$$
\mathcal{A}=G_{1} \times{ }_{2}^{1} \mathcal{G}_{2} \times{ }_{3}^{1} \mathcal{G}_{3} \times{ }_{3}^{1} \cdots \times{ }_{3}^{1} \mathcal{G}_{N-1} \times{ }_{3}^{1} G_{N} .
$$

Thus, person $p$ in a specific combination of all the other $(N-3)$ features can be expressed as

$$
\mathcal{A}\left(:,:, i_{3}, \cdots, i_{N-1}, p\right)=G_{1} \times{ }_{2}^{1} \mathcal{G}_{2} \times{ }_{3}^{1} \mathcal{G}_{3}^{\left(i_{3}\right)} \times{ }_{3}^{1} \cdots \times{ }_{3}^{1} \mathcal{G}_{N-1}^{\left(i_{N-1}\right)} \times{ }_{3}^{1} G_{N}(:, p) .
$$

Let $C^{\left(i_{3}, \cdots, i_{N-1}\right)}=G_{1} \times \frac{1}{2} \mathcal{G}_{2} \times{ }_{3}^{1} \mathcal{G}_{3}^{\left(i_{3}\right)} \times{ }_{3}^{1} \cdots \times{ }_{3}^{1} \mathcal{G}_{N-1}^{\left(i_{N-1}\right)}$. Then, (5.2) becomes

$$
\mathcal{A}\left(:,:, i_{3}, \cdots, i_{N-1}, p\right)=C^{\left(i_{3}, \cdots, i_{N-1}\right)} \times_{3} g_{N}^{(p)},
$$

where $g_{N}^{(p)}=G_{N}(:, p)$.

Given an image vector $z \in \mathbb{R}^{n_{1} n_{2}}$ of an unknown person in an unknown combination of the $(N-3)$ features, we define the distance between $z$ and the person $p$ as

$$
D_{T T N}\left(i_{3}, \cdots, i_{N-1}, p\right)=\left\|\hat{\alpha}_{i_{3}, \cdots, i_{N-1}}-g_{N}^{(p)}\right\|,
$$

where

$$
\hat{\alpha}_{i_{3}, \cdots, i_{N-1}}=\operatorname{argmin}_{\alpha_{i_{3}, \cdots, i_{N-1}}}\left\|\operatorname{unfold}\left(C^{\left(i_{3}, \cdots, i_{N-1}\right)}\right) \alpha_{i_{3}, \cdots, i_{N-1}}-z\right\| .
$$

6. Description of the Databases. In the following we consider a database of faces of $n_{p}$ persons in $n_{e}$ different expressions - where by expression we possibly mean different illuminations, view angle, etc. - with $n_{i}=n_{1} n_{2}$ pixels. Each image can be either considered as an $n_{1} \times n_{2}$ matrix, or as a $n_{1} n_{2}$ vector, so that such a database can be represented either by a 4 -way tensor $\mathcal{A} \in \mathbb{R}^{n_{1} \times n_{2} \times n_{e} \times n_{p}}$ or by a 3 -way tensor $\mathcal{A} \in \mathbb{R}^{n_{i} \times n_{e} \times n_{p}}$.

In this work we consider eight different datasets. For the first four ones it holds that $n_{i}<n_{e}$, while for the others $n_{i}>n_{e}$. This selection was performed to computationally illustrate the need to use tensor strategies whenever the number of pixel is larger than the number of expressions, to be able to classify a new image with a high success rate.

1. The Orl database, which contains 400 grayscale images in PGM format of 40 persons. Each subject is photographed in 10 different expressions. In Figure 6.1 all the expressions of subject 1 are shown.

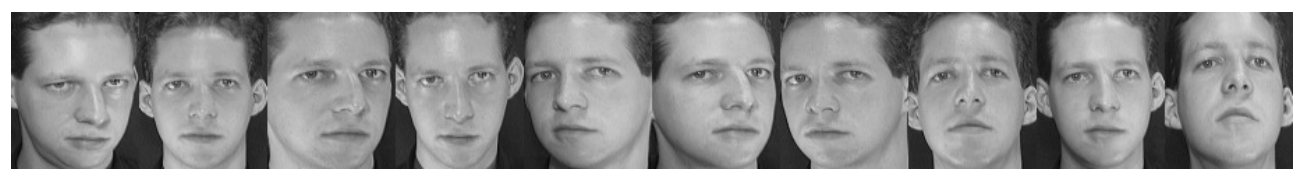

Fig. 6.1: Subject 1 of the Orl database.

2. The Faces95 database, which consists of 1440 RGB images in JPG format of 72 persons in slightly different positions with respect to the camera for a total of 20 expressions. RGB images were transformed in gray images within Matlab. The same was done for the other images with colors. In Figure 6.2 the first subject is reported in all expressions. 


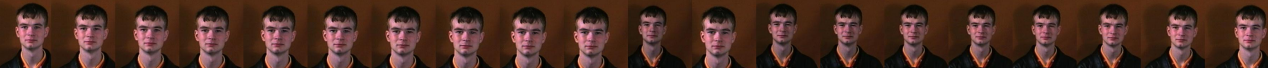

Fig. 6.2: Subject 1 of the Faces95 database photographed in 20 different expressions.

3. The Faces96 database, which is composed by 2261 images in JPG format of 119 persons at different distance with respect to the camera totalling 19 expressions. In Figure 6.3 the first subject is reported in all the expressions.

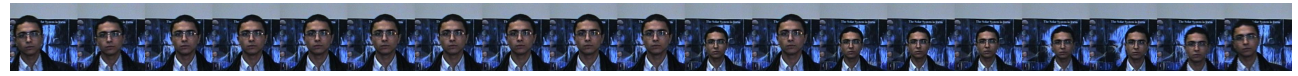

Fig. 6.3: Subject 1 of the Faces96 database.

4. The COIL-20 database composed by 20 objects, each of which is photographed in 72 different view angles. In Figure 6.4 object 1 in 15 different view angles is shown.

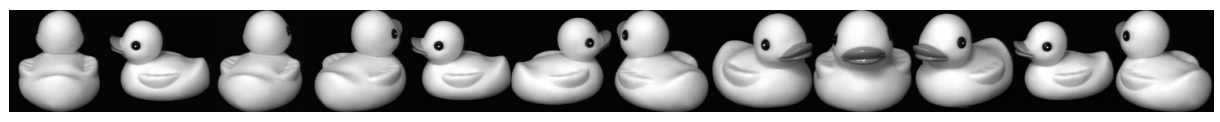

Fig. 6.4: Object 1 of the COIL-20 database.

5. The Extended Yale database, which contains 16380 grayscale images in PGM format of 28 persons. Each person is photographed in 9 poses and 65 illumination conditions for a total of 585 expressions. A shrunk version ${ }^{3}$ of the Extended Yale database is used. In Figure 6.5 subject 1 is reported in 15 different expressions.

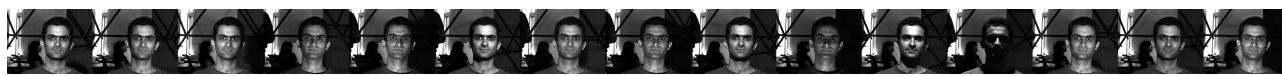

Fig. 6.5: Subject 1 of the Extended Yale database in 15 different expressions.

6. The MIT-CBCL database, which contains 3240 grayscale images of 10 persons in 324 different expressions. ${ }^{4}$ The original pixel size of each image is $200 \times 200$. For our experiments the pixel size is reduced to $15 \times 15$. In Figure 6.6, 10 different expressions of person one are shown.

7. The MNIST database, from which we selected about 1901 grayscale $28 \times 28$ images of each of the 10 handwritten digits. In fact, for each digit we used 260 images as test set, and more images as training set, whose number depends on the used test-training splitting percentage. For this dataset the third variable cannot be given the interpretation of "expression". Indeed, there is no

\footnotetext{
${ }^{3}$ Each image has been reduced to the pixel size $20 \times 15$, instead of the original $640 \times 480$ size.

${ }^{4}$ In our experiments only the training set of the database is considered for generating the training and test phases.
} 


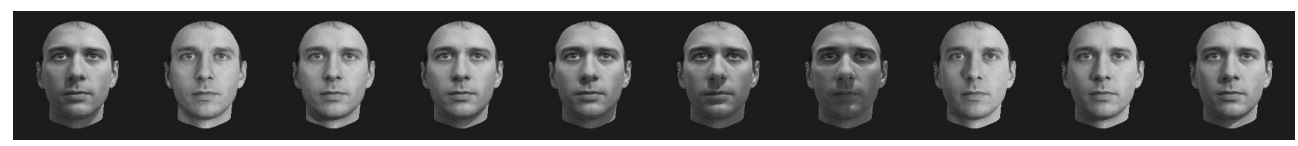

Fig. 6.6: Subject 1 of the MIT-CBCL database in 10 different expressions.

explicit correspondence between the same $j$ th image of two different digits, so that images of each digit occur in a completely random order, though they all correspond to some sorts of stretching of a reference digit. We shall refer to this as a lack of a classification feature. In Figure 6.7, 10 different versions of the digit "three" are shown.

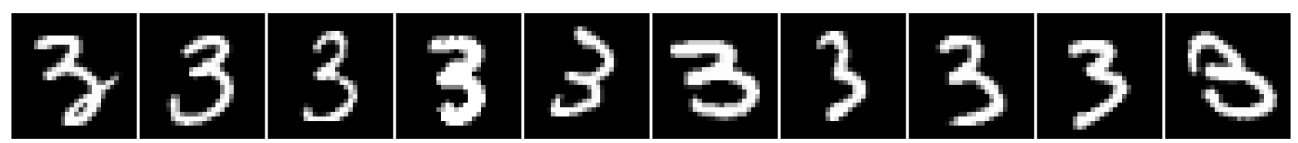

Fig. 6.7: Digit 3 of the MNIST database.

8. The Fashion MNIST database, which contains 70000 images of 10 different kinds of Zalando's articles. The Dataset is split in a training set, composed by 6000 images, and a test set, composed by 1000 images. For our experiments only a subset of the whole dataset is used. In particular we use all the images of the test set but only a subset of images of the training set, as required by the chosen test-training splitting percentage. In Figure 6.8 the first object is represented in 10 different conditions. Notice that, as for the MNIST, the variable expression is not defined, thus this variable lacks of a classification feature.

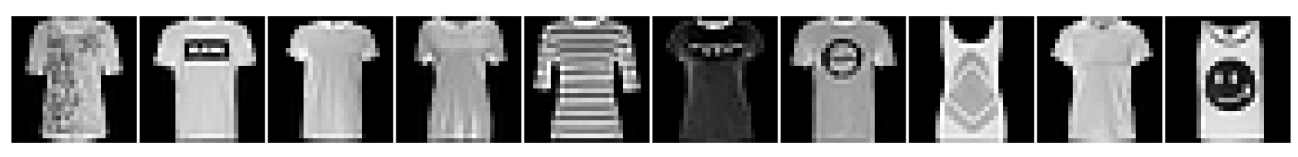

Fig. 6.8: First object of the Fashion MNIST database.

The characteristics of all databases are summarized in Table 6.1.

7. Numerical experiments. The validation of a classification algorithm needs to be addressed by using different perspectives. The most obvious way to evaluate the algorithm performance is to inspect its median success rate across several trials, as we are going to show in Table 7.1. However, other important measures should be taken into account to perform a fair comparison among different classification strategies. For example, $\mathrm{CPU}$ time, memory requirements and statistical classification parameters may be crucial in ranking the given methods, depending on the application where the algorithm is supposed to be used. For these reasons, in the next two subsections we report on our computational experience using the mentioned performance measures. 


\begin{tabular}{|c|c|c|c|c|}
\hline Database & pixel size & pixel size (vector format) & $n_{e}$ & $n_{p}$ \\
\hline Orl & $92 \times 112$ & 10304 & 10 & 40 \\
\hline COIL-20 & $128 \times 128$ & 16384 & 72 & 20 \\
\hline Faces95 & $180 \times 200$ & 36000 & 20 & 72 \\
\hline Faces96 & $196 \times 196$ & 38416 & 19 & 119 \\
\hline Ext'd Yale shrunk & $20 \times 15$ & 300 & 585 & 28 \\
\hline MNIST & $28 \times 28$ & 784 & 1901 & 10 \\
\hline Fashion MNIST & $28 \times 28$ & 784 & 7000 & 10 \\
\hline MIT-CBCL & $15 \times 15$ & 225 & 324 & 10 \\
\hline
\end{tabular}

Table 6.1: Pixel size, number of expressions and persons of all databases.

7.1. A first Classification test. In this section we first report on our numerical experiments where we use the success rate as performance measure. To this end, we split each database in a training set and a test set. The split is made by taking the $s \%$ of the $n_{e}$ expressions for each person as training set and the remaining ones as test set. The expressions used as training set are chosen randomly. We report results for $s=80 \%, 50 \%$; the tensor methods were not overly sensitive to the choice of this parameter. In Table 7.1 we report the success rate of all considered algorithms, namely the matrix SVD, the tensor SVD (HOSVD), the three- and four-dimensional TT (TT 3D and TT 4D) and the simple least squares (LS) methods. For all datasets except the large MNIST ones, all percentages correspond to the average of 20 consecutive runs.

\begin{tabular}{|c|c|c|c|c|c|c|}
\hline$s \%$ & Database & SVD & HOSVD & TT 3D & TT 4D & LS \\
\hline \multirow{4}{*}{80} & Orl & $\mathbf{9 7 . 4 2} \%$ & $94.44 \%$ & $96.65 \%$ & $96.69 \%$ & $95.81 \%$ \\
\cline { 2 - 7 } & COIL-20 & $\mathbf{9 9 . 9 5} \%$ & $97.50 \%$ & $99.35 \%$ & $99.35 \%$ & $99.17 \%$ \\
\cline { 2 - 7 } & Faces95 & $90.00 \%$ & $86.56 \%$ & $87.15 \%$ & $\mathbf{9 1 . 0 3} \%$ & $86.44 \%$ \\
\cline { 2 - 7 } & Faces96 & $100 \%$ & $97.68 \%$ & $\mathbf{1 0 0} \%$ & $98.50 \%$ & $97.23 \%$ \\
\cline { 2 - 7 } & Ext'd Yale shrunk & $59.59 \%$ & $99.08 \%$ & $99.24 \%$ & $98.72 \%$ & $\mathbf{9 9 . 7 8} \%$ \\
\cline { 2 - 7 } & MNIST & $62.55 \%$ & $92.27 \%$ & $91.05 \%$ & $91.03 \%$ & $\mathbf{9 2 . 6 9} \%$ \\
\cline { 2 - 7 } & Fashion MNIST & $49.19 \%$ & $78.63 \%$ & $79.20 \%$ & $77.74 \%$ & $\mathbf{8 4 . 7 6} \%$ \\
\cline { 2 - 7 } 50 & MIT-CBCL & $23.52 \%$ & $100 \%$ & $\mathbf{1 0 0} \%$ & $100 \%$ & $100 \%$ \\
\hline \multirow{4}{*}{} & Orl & $\mathbf{9 4 . 8 4} \%$ & $91.38 \%$ & $92.96 \%$ & $92.75 \%$ & $89.93 \%$ \\
\cline { 2 - 7 } & COIL-20 & $\mathbf{9 9 . 8 4} \%$ & $96.10 \%$ & $98.90 \%$ & $96.71 \%$ & $98.04 \%$ \\
\cline { 2 - 7 } & Faces95 & $96.25 \%$ & $\mathbf{9 6 . 2 5} \%$ & $83.90 \%$ & $87.50 \%$ & $80.24 \%$ \\
\cline { 2 - 7 } & Eaces96 & $98.66 \%$ & $97.34 \%$ & $97.94 \%$ & $\mathbf{9 9 . 3 3} \%$ & $96.87 \%$ \\
\cline { 2 - 7 } & Ext'd Yale shrunk & $\mathbf{9 9 . 8 7} \%$ & $98.70 \%$ & $98.74 \%$ & $98.99 \%$ & $99.64 \%$ \\
\cline { 2 - 7 } & MNIST & $71.25 \%$ & $\mathbf{9 0 . 8 7} \%$ & $89.12 \%$ & $89.12 \%$ & $89.83 \%$ \\
\cline { 2 - 7 } & Fashion MNIST & $40.34 \%$ & $74.58 \%$ & $75.22 \%$ & $74.57 \%$ & $\mathbf{8 1 . 3 1 \%}$ \\
\cline { 2 - 7 } & MIT-CBCL & $100 \%$ & $100 \%$ & $\mathbf{1 0 0} \%$ & $100 \%$ & $100 \%$ \\
\hline
\end{tabular}

Table 7.1: Success rate (as percentage) of all classification algorithms.

Table 7.1 shows the SVD-based algorithm works well only when $n_{i}>n_{e}$,i.e. with the Orl, the COIL-20, the Faces95 and the Faces96 datasets. However, in many applications it happens that $n_{i}<n_{e}$ because of the huge number of features available. 
This suggests that alternative strategies should be considered whenever the number of, say, expressions $n_{e}$ is significantly larger than the number of pixels $n_{i}$. As we can see from Table 7.1, tensor methods provide viable strategies. It is also interesting to observe that LS works quite well for the datasets where the third variable does not provide a real classification feature, that is for the two MNIST sets.

Memory requirements of the tensor methods can be quite different. Given the tensor $\mathcal{A} \in \mathbb{R}^{n_{i} \times n_{e} \times n_{p}}$, the following table summarizes the minimum storage requirements:

\begin{tabular}{ll} 
Method & Memory allocations \\
\hline HOSVD & $n_{i} n_{e} n_{p}+n_{i}^{2}+n_{e}^{2}+n_{p}^{2}$ \\
TT 3D & $n_{i}^{2}+n_{i} n_{e} n_{p}+n_{p}^{2}$ \\
\hline
\end{tabular}

In particular, HOSVD requires to store the core tensor $\mathcal{S} \in \mathbb{R}^{n_{i} \times n_{e} \times n_{p}}$ and three orthonormal matrices $U^{(1)} \in \mathbb{R}^{n_{i} \times n_{i}}, U^{(2)} \in \mathbb{R}^{n_{e} \times n_{e}}$ and $U^{(3)} \in \mathbb{R}^{n_{p} \times n_{p}}$. On the other hand, TT 3D needs to store $G_{1} \in \mathbb{R}^{n_{i} \times n_{i}}, \mathcal{G}_{2} \in \mathbb{R}^{n_{i} \times n_{e} \times n_{p}}$ and $G_{3} \in \mathbb{R}^{n_{p} \times n_{p}}$, thus leading to lower memory requirements. Truncation strategies additionally decrease the memory demand of the tensor-train method.

In this work, we considered only two features for each person, but in realistic applications a higher number of features may be available. In such situations the Tensor-Train format enables one to store only third order tensors, whereas the HOSVD requires to allocate memory for a core tensor with the same order of $\mathcal{A}$.

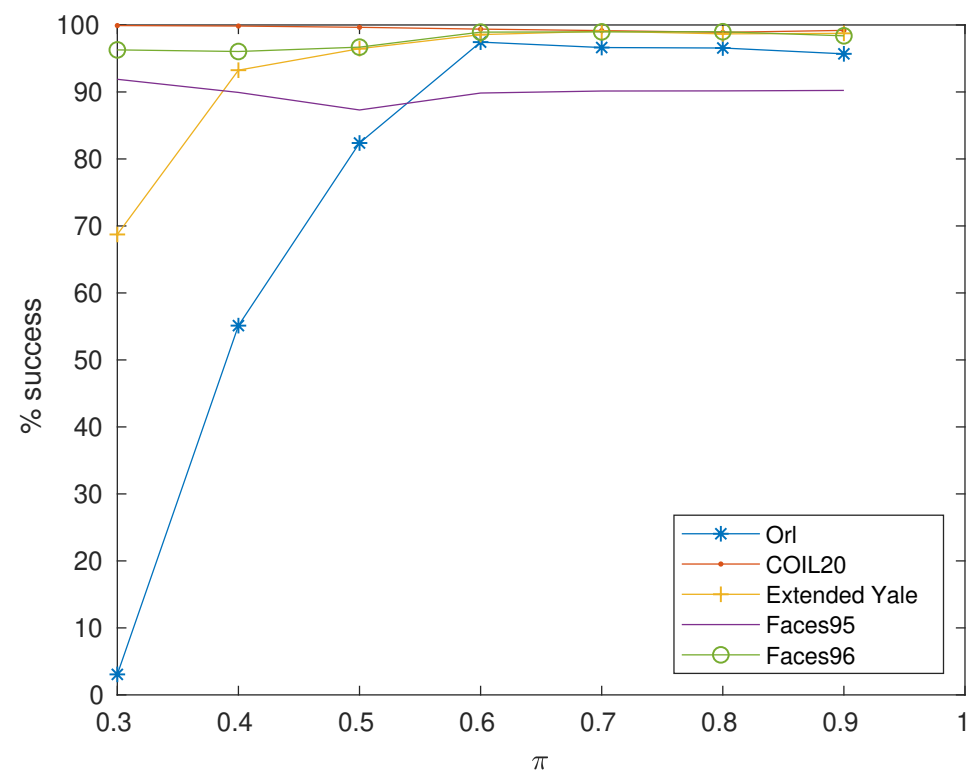

Fig. 7.1: Percentage of success of the truncated TT 3D, for different values of the truncation parameter $\pi$, tested on different databases.

Figure 7.1 shows the percentage of success of the truncated procedure on five databases, when different values of the truncation threshold $\pi$ are used (see section 3). The higher efficiency in terms of computational costs (CPU time) and mem- 
ory requirements together with the good recognition success are all in favor of the algorithm truncated version, instead of the full one. For instance, for $\pi=0.9$ in the Extended Yale Database only $32 \%$ of the singular values are retained, making the memory requirements of the truncated version significantly lower than those of the non-truncated one. As we can see from Figure 7.1 a value of $\pi=0.6$ enables us to reach good classification performance for almost all databases.

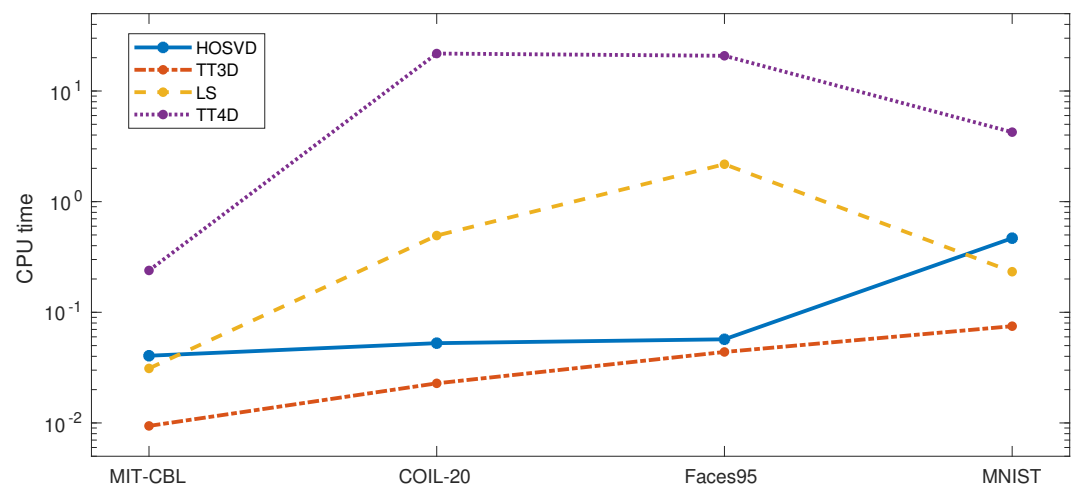

Fig. 7.2: CPU time (secs) required for the classification of a person $p$ in a specific expression $e$.

Figure 7.2 reports on the computational costs of the tensor-based methods on the four most time consuming datasets; for the very large dataset Fashion-MNIST runs were performed on a different computer and therefore they are not reported. The reported CPU time (in logarithmic scale) is the time required for the classification of a person $p$, in an expression $e$ whenever appropriate. The plot shows that the CPU time of TT 3D is lower than for all other tensor-based methods. Hence, this performance measure can help discriminate among methods whenever the classification success rate and memory requirements are comparable.

7.2. Performance using statistical classification measures. Using an applied statistics terminology, the Face Recognition problem can be thought of as a multiclass classification problem, where the classes are the different persons of a specific database. For a database of $n_{p}$ persons in $n_{e}$ different expressions, we consider the splitting in a training set ( $n_{p}$ persons in $0.75 n_{e}$ expressions) and a test set $\left(n_{p}\right.$ persons in $0.25 n_{e}$ expressions). After the classification of all images in the test set has been completed, for each person $p$ the following quantities can be computed, giving rise to the so-called "confusion matrix":

- true positive $\left(t p_{p}\right)$ : number of subjects correctly classified as person $p$;

- true negative $\left(t n_{p}\right)$ : number of correctly recognized subjects that are not person $p$

- false positive $\left(f p_{p}\right)$ : number of subjects incorrectly classified as person $p$;

- false negative $\left(f n_{p}\right)$ : number of subjects not recognized as person $p$. 
Using these four quantities, the following classifier parameters can be computed:

$$
\begin{aligned}
& \text { Accuracy }=\frac{1}{n_{p}} \sum_{p=1}^{n_{p}} \frac{t p_{p}+t n_{p}}{t p_{p}+t n_{p}+f p_{p}+f n_{p}}, \quad \text { Precision }=\frac{1}{n_{p}} \sum_{p=1}^{n_{p}} \frac{t p_{p}}{t p_{p}+f p_{p}}, \\
& \text { Recall }=\frac{1}{n_{p}} \sum_{p=1}^{n_{p}} \frac{t p_{p}}{t p_{p}+f n_{p}},
\end{aligned}
$$

These parameters provide a measure of reliability of the employed algorithm: the closer the parameter is to $100 \%$ the more robust the corresponding classification strategy is. Depending on the realistic application for which we want to use the classification algorithm, one can be interested in maximizing one of these three quantities. For example, in situations such as border controls or medical tests, one may be interested in maximizing the recall, since this means minimizing the number of images of person $p$ that are not recognized as person $p$.

In Table 7.2 precision, accuracy and recall are displayed, using macro-averaging (for further details see [16]). The results show an overall different ranking of the methods when these measures are considered, with respect to the percentages in Table 7.1. HOSVD appears to be the most reliable strategy for all three parameters for the MNIST database, whereas for all other datasets tensor-train based algorithms have better performance. It is also interesting that, although the three parameters measure truly different things, the relative ranking of all algorithms does not change much going from, say, Accuracy to Recall.

\begin{tabular}{|c|c|c|c|c|c|c|c|c|}
\hline \multirow{2}{*}{ measure } & Orl & $\begin{array}{c}\text { COIL } \\
-20\end{array}$ & Faces95 & Faces96 & $\begin{array}{c}\text { Ext'd } \\
\text { Yale }\end{array}$ & MNIST & $\begin{array}{c}\text { Fashion } \\
\text { MNIST }\end{array}$ \\
\hline \multirow{3}{*}{ Accuracy } & HOSVD & $99.71 \%$ & $\mathbf{9 9 . 7 7} \%$ & $99.66 \%$ & $99.97 \%$ & $99.93 \%$ & $\mathbf{9 8 . 1 8} \%$ & $95.26 \%$ \\
\cline { 2 - 9 } & TT 3D & $\mathbf{9 9 . 7 8} \%$ & $\mathbf{9 9 . 7 7} \%$ & $\mathbf{9 9 . 7 1} \%$ & $\mathbf{9 9 . 9 8} \%$ & $\mathbf{9 9 . 9 4} \%$ & $97.83 \%$ & $\mathbf{9 5 . 3 9} \%$ \\
\cline { 2 - 9 } Precision & TT 4D & $99.77 \%$ & $\mathbf{9 9 . 7 7} \%$ & $99.60 \%$ & $\mathbf{9 9 . 9 8} \%$ & $99.94 \%$ & $97.67 \%$ & $\mathbf{9 5 . 3 9} \%$ \\
\cline { 2 - 9 } & HOSVD & $76.62 \%$ & $\mathbf{9 8 . 0 3} \%$ & $91.10 \%$ & $98.60 \%$ & $99.06 \%$ & $\mathbf{9 1 . 1 8} \%$ & $78.30 \%$ \\
\cline { 2 - 8 } & TT 3D & $\mathbf{9 6 . 7 3} \%$ & $\mathbf{9 8 . 0 3} \%$ & $\mathbf{9 2 . 5 3} \%$ & $99.07 \%$ & $99.17 \%$ & $89.89 \%$ & $\mathbf{7 8 . 5 3} \%$ \\
\cline { 2 - 9 } Recall & TT 4D & $96.58 \%$ & $\mathbf{9 8 . 0 3} \%$ & $90.01 \%$ & $\mathbf{9 9 . 2 0} \%$ & $\mathbf{9 9 . 2 0} \%$ & $89.00 \%$ & $\mathbf{7 8 . 5 3} \%$ \\
\cline { 2 - 9 } & HOSVD & $94.29 \%$ & $97.67 \%$ & $87.67 \%$ & $98.29 \%$ & $99.01 \%$ & $\mathbf{9 0 . 9 0} \%$ & $76.31 \%$ \\
\cline { 2 - 9 } & TT 3D & $\mathbf{9 5 . 6 2} \%$ & $\mathbf{9 7 . 6 9} \%$ & $\mathbf{8 9 . 6 0} \%$ & $98.85 \%$ & $99.12 \%$ & $89.10 \%$ & $\mathbf{7 6 . 9 3} \%$ \\
\cline { 2 - 9 } & TT 4D & $95.50 \%$ & $\mathbf{9 7 . 6 9} \%$ & $85.62 \%$ & $\mathbf{9 9 . 0 1} \%$ & $\mathbf{9 9 . 1 6} \%$ & $88.32 \%$ & $\mathbf{7 6 . 9 3} \%$ \\
\hline
\end{tabular}

Table 7.2: Accuracy, precision and recall for the tensor-based classification algorithms.

8. Conclusions and perspectives. Based on the Tensor-Train decomposition of the given dataset, we have proposed a new algebraic strategy for face and object recognition, both in a three-dimensional and four-dimensional setting. The use of the TT form allows one to easily treat truncation, which reduces both CPU time and memory requirements, without sacrificing the recognition success rate. Moreover, the TT-based algorithm is preferable to HOSVD since it does not suffer from the course of dimensionality, naturally extending to more than three dimensions, thus allowing for the inclusion of additional features as extra dimensions. Indeed, although we limited our exploration of multiple dimensions to splitting image pixels, depending on the application different characteristic features could be available for each person. For face recognition, for instance, different age or backdrop image sets could determine additional dimensions. 
Our computational experiments on eight different datasets seem to show that using the Tensor-Train form allows one to achieve good classification success for comparable memory requirements (in the full case) and smaller CPU time with respect to the now classical tensor based HOSVD. Due to the relatively low computational costs of the TT methodology, we also envision using the proposed classification algorithm as a preliminary first pass to a significantly more expensive but even more accurate procedure, e.g., within deep learning methodology, to narrow the classification dataset.

9. Acknowledgements. Both authors are members of the GNCS-Indam activity group. Its financial support is fully acknowledged.

\section{REFERENCES}

[1] Brett W. Bader, Tamara G. Kolda, et al. Matlab tensor toolbox version 3.0-dev. Available online, October 2017.

[2] M. Boussé, N. Vervliet, O. Debals, and L. De Lathauwer. Face recognition as Kronecker product equation. IEEE International Workshop on Computational Advances in MultiSensor Adaptive Processing (CAMSAP), 2017.

[3] A. Cichocki, D. Mandic, A-H. Phan, C. Caiafa, G. Zhou, Q. Zhao, and L. De Lathauwer. Tensor decompositions for signal processing applications from two-way to multiway component analysis, 2014.

[4] Andrzej Cichocki. Era of big data processing: A new approach via tensor networks and tensor decompositions. CoRR, abs/1403.2048, 2014.

[5] Lieven De Lathauwer, Bart De Moor, and Joos Vandewalle. A multilinear singular value decomposition. SIAM journal on Matrix Analysis and Applications, 21(4):1253-1278, 2000.

[6] Kresimir Delac, Mislav Grgic, and Marian Stewart Bartlett, editors. Recent Advances in Face Recognition. In-Teh, 2008.

[7] Lars Eldén. Matrix methods in data mining and pattern recognition, volume 4. SIAM, 2007.

[8] Gene H Golub and Charles F Van Loan. Matrix computations. JHU press, 3rd edition, 2012.

[9] Ning Hao, Misha E Kilmer, Karen Braman, and Randy C Hoover. Facial recognition using tensor-tensor decompositions. SIAM Journal on Imaging Sciences, 6(1):437-463, 2013.

[10] Tamara G Kolda and Brett W Bader. Tensor decompositions and applications. SIAM review, 51(3):455-500, 2009.

[11] Sebastian Krämer. A geometric description of feasible singular values in the Tensor Train format, 2017.

[12] Alexander Novikov, Dmitry Podoprikhin, Anton Osokin, and Dmitry P. Vetrov. Tensorizing neural networks. CoRR, abs/1509.06569, 2015.

[13] Ivan Oseledets. Tensor-train decomposition. SIAM Journal on Scientific Computing, 33(5):2295-2317, 2011.

[14] Ivan Oseledets and Eugene Tyrtyshnikov. TT-cross approximation for multidimensional arrays. Linear Algebra and its Applications, 432(1):70-88, 2010.

[15] Andrew W. Senior and Ruud M. Bolle. Face recognition and its application. In David Zhang, editor, Biometric Solutions: For Authentication in an E-World, pages 83-97. Springer US, Boston, MA, 2002.

[16] M. Sokolova and G. Lapalme. A systematic analysis of performance measures for classification tasks. Information Processing and Management, 45:427-437, 2009.

[17] Daniel Tock. Tensor decomposition and its applications. Master's thesis, University of Chester, 2010.

[18] M.A.O. Vasilescu and D. Terzopoulos. Multilinear analysis of image ensembles: Tensorfaces. In Heyden A., Sparr G., Nielsen M., and Johansen P., editors, European Conference on Computer Vision, volume 2350 of Lecture Notes in Computer Science, pages 447-460. Springer, Berlin, Heidelberg, 2002.

[19] Yinchong Yang, Denis Krompass, and Volker Tresp. Tensor-train recurrent neural networks for video classification. CoRR, abs/1707.01786, 2017. 